\title{
Effectiveness of Early Physiotherapy Interventions for Weight Gain in Low Birth Weight Neonates
}

\author{
Pratik Goykar ${ }^{1}$ and Namrata Kadam²* \\ 'Intern Physiotherapy, Krishna College of Physiotherapy, Krishna Institute of Medical Sciences, Karad - 415110, \\ Maharashtra, India \\ ${ }^{2}$ Assistant Professor, Department of Pediatrics Physiotherapy, Krishna College of Physiotherapy, Krishna Institute of Medical \\ Sciences, Karad - 415110, Maharashtra, India; dr.namratakcpt@gmail.com
}

\begin{abstract}
Background: Prematurity is a leading cause of death in Indian scenario. There are lack of studies done on early physiotherapy interventions in neonates. Early care interventions in premature newborns show positive effect. Growth retardation and demineralisation of bones in newborn are thought to be caused by prolong immobilisation or lack of physical activities. In this study we are going to give combination of massage, passive movements, facilitatory techniques, joint compression and kangaroo mother care, which has not been done before. Indeed this made us to study the effectiveness of early physiotherapy intervention on weight gain in low birth weight neonates. Objectives: To determine the effectiveness of early physiotherapy intervention on weight gain in moderate premature neonates (32-37 weeks), very premature (28-32 weeks) and extremely premature neonates (less than 28 weeks). Methods: Individuals fulfilling the inclusive criteria were selected. Written consent was taken from parents and care taker of neonates. Total 20 low birth weight neonates were taken. They were differentiated into moderate premature, very premature and extremely premature neonates. The weight was measured and breast feeding frequency was noted before treatment. Then the neonates were given massage, passive movements, proprioception stimulation by individual joint compression, oral stimulation and kangaroo mother care. This was given for 25-30 minutes each session for two times per day for 7 days. After that again weight and breast feeding frequency was checked to see progression. Later evaluation and interpretation was done using statistical analysis. Results: The early physiotherapy intervention is effective in low birth weight baby for improving weight in pre mature neonates. Conclusion: On the basis of result it can be concluded that the early physiotherapy is effective in improving weight, breast feeding frequency in low birth weight neonates. Hence, early physiotherapy interventions should be considered to decrease the morbidity and mortality rate of LBW neonates.
\end{abstract}

Keywords: Breast Feeding, Early Physiotherapy, Neonatal Intensive Care Unit (NICU), Physiotherapy Intervention, Premature, Weight Gain

\section{Introduction}

Preterm birth complication's account for $35 \%$ of all neonatal deaths and constitute the most important cause of neonatal mortality ${ }^{1}$.

Preterm birth (premature birth) is a significant public health problem across the world due to associated neonatal (first 28 days of life) mortality and short-term and long-term morbidity and disability in later life.

Preterm is defined as babies born alive before 37 weeks of pregnancy is completed ${ }^{2}$. There are sub-categories of preterm birth, based on gestational age:

- Extremely preterm (less than 28 weeks)

- Very preterm (28 to 32 weeks)

- Moderate to late preterm (32 to 37 weeks)
Low Birth Weight (LBW) is defined as a birth weight less than 2500 grams irrespective of gestational age ${ }^{2}$. Early physiotherapy intervention on weight gain in low birth weight babies is useful for better outcome in weight gain of the preterm babies. This can reduce the Neonatal Intensive Care Unit (NICU) stay of the newborn baby with faster recovery and better outcome. There are different techniques in physiotherapy for weight gain in low birth weight babies like

- Massage

- Passive movements

- Proprioceptive stimulation

- Oral stimulation

- Kangaroo mother care

*Author for correspondence 
Massage is stated as a mechanical manipulation of body tissues with rhythmical pressure and stroking for the purpose of promoting health ${ }^{3,4}$. In most of the studies done on preterm infant massage studies have combined tactile and kinesthetic stimulation which have shown increased weight gain due to stimulation of baroreceptors and mechanoreceptors which can increase vagal activities. ${ }^{5}$. The techniques such as kinesthetic stimulation are also effective in reducing pain and preventing neuromuscular complications ${ }^{6}$. Massage therapy is referred as touch intended to stimulate the baby. Massage is also a facilitatory technique. Number of studies has shown massage to increase development of sympathetic nervous system and help in neuromuscular development ${ }^{2}$.

Due to prolong immobilisation of neonates in NICU, their bone mass density may hamper. Due to daily passive range of movements their bone mass density can improve? ${ }^{7}$.

Kangaroo Mother Care (KMC) is defined as early, prolong and continuous skin to skin contact between the mother and preterm LBW baby $^{2}$. Kangaroo mother care improves weight gain in preterm and low birth weight babies and also helps in reducing pain ${ }^{3,6}$. KMC results in keeping neonates warm and protect them against cold stress and hypothermia. KMC significantly increases milk production in mothers and there by result in increased breast feeding rate. It also improves weight in low birth weight infants and it also helps in emotional bonding between mother and infant which can result in early discharge from the hospital ${ }^{8}$.

Proprioceptive stimulation is provided through joint compression to facilitate normal movement patterns during therapeutic handling'.

It also helps to facilitate movement, improve muscle tone and assist in motor organization ${ }^{10}$. Normalization of tone helps to improve active movements and coordinated movements which results in to better activity.

Oral stimulations help to improve the sucking in preterm neonates.

Many studies have shown the use of only one or two individual techniques to improve the weight gain in LBW neonates. In this study we are going to give combination of all this intervention to see the early effect on weight gain in LBW neonates.

\section{Materials and Methods}

This was a study to find the effectiveness of early physiotherapy intervention on weight gain in low birth weight neonates. The study was carried out in Krishna hospital, Karad. An approval for the study was obtained from the protocol committee and ethical committee of KIMSDU. Individuals were approached and those fulfilling the inclusive criteria were selected. The purpose was explained and written inform consent was taken from parents and care taker of neonates prepared in accordance with the Helsinki declaration from those who were willing to participate. Total $20 \mathrm{LBW}$ neonates were taken. The inclusion criteria was extremely preterm neonates (less than 28 weeks), very preterm neonates ( 28 to 32 weeks), moderate to late preterm neonates ( 32 to 37 weeks) and babies who were hemodynamically stable and babies who have completed 10 days of extra uterine life. The exclusion criteria was full term neonates with LBW, full term neonates with normal birth weight, major congenital anomalies and inability to undergo intervention (any fracture) or perinatal asphyxia. The weight of neonates were assessed by digital weighing machine, the breast feeding frequency was noted. The neonates were given massage, passive movements, proprioception stimulation by individual joint compression, oral stimulation was given to checks, lips and gums by sterile hand gloves and by gentle stroking to checks starting from base of nose to ears and back to angle of lips. Circular strokes around the lips and strokes going from forward to backward direction to both upper and lower gums and kangaroo mother care. This was given for 25-30 minutes each session for two times per day for 7 days. After that again weight and breast feeding frequency was checked to see progression. Later evaluation and interpretation was done using statistical analysis.

\section{Statistical Analysis}

The unpaired $t$ test for weight measurement and breast feeding frequency was used for analysis of data. Statistical analysis of the recorded data was done by using the software SPSS version 20. The p value is less than 0.0001 which is extremely significant for pre and post treatment breast feeding frequency.

\section{Results}

There is a significant difference between pre and post treatment outcome measures. The more improvement is seen in moderately preterm neonates for weight gain and there was no significant difference found in comparison of post treatment breast feeding frequencies in between moderately, very extremely preterm neonates. Hence, the early physiotherapy intervention is effective in low birth weight baby for improving weight in pre mature neonates (Graph 1).

\subsection{Interpretation}

In this Graph 1 there is comparison of pre treatment weight and post treatment weight. It shows that the post treatment weight is increased and there is significant difference between pre and post treatment weight. 


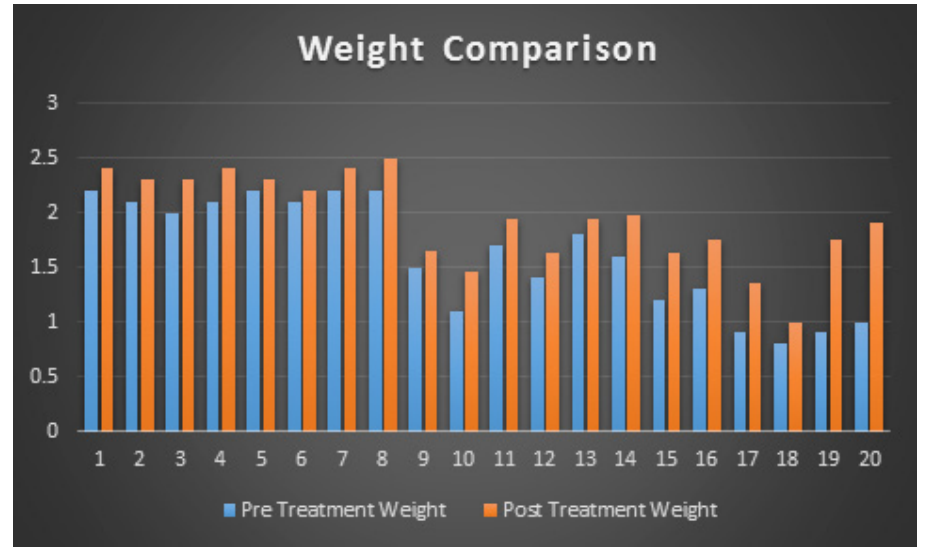

Graph 1. Comparision of weight.

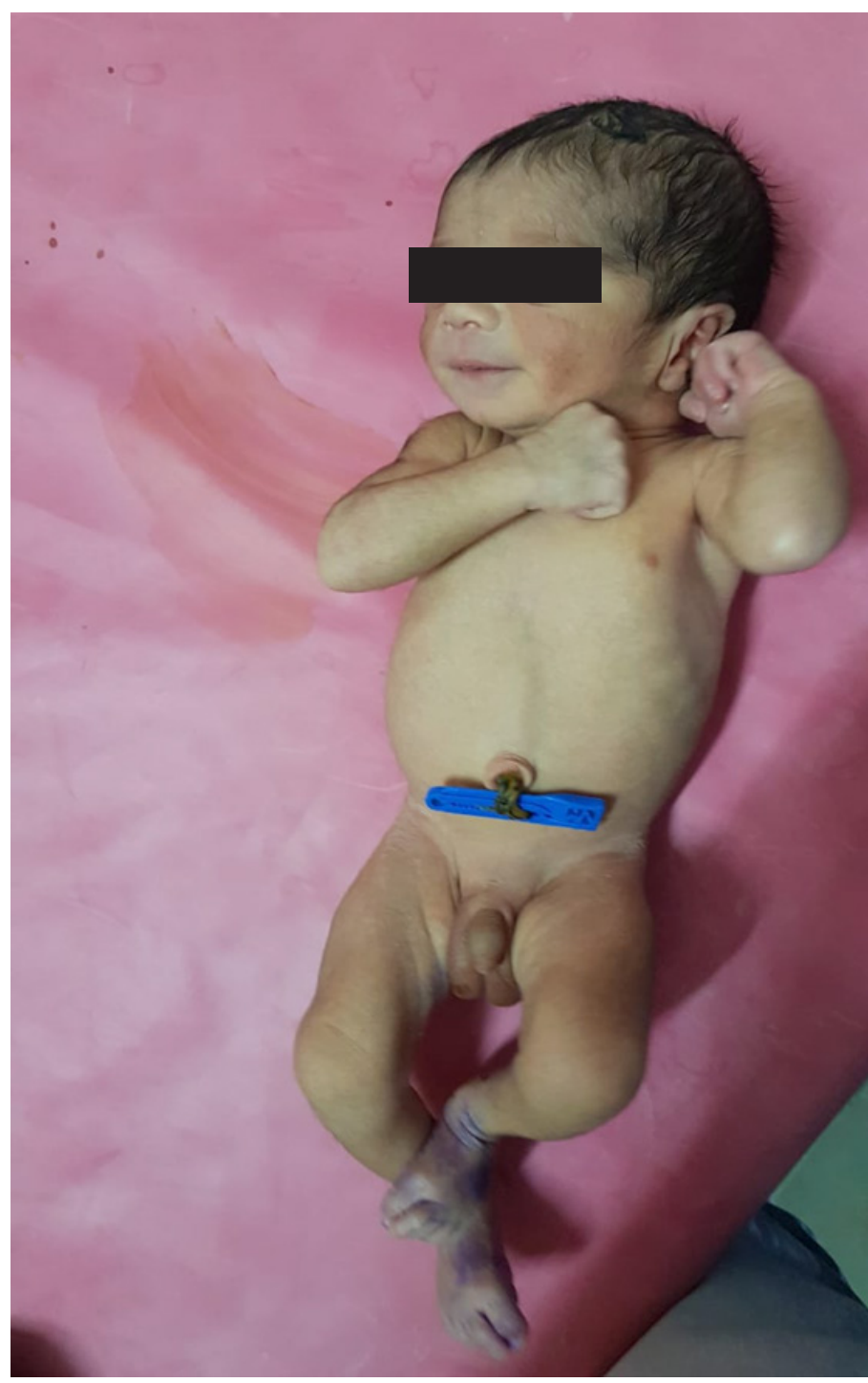

\subsection{Interpretation}

In this Graph 2 there is comparison of breast feeding frequency pre and post treatment per day. It shows that the post treatment breast frequency is increased by 2-3 times per day.

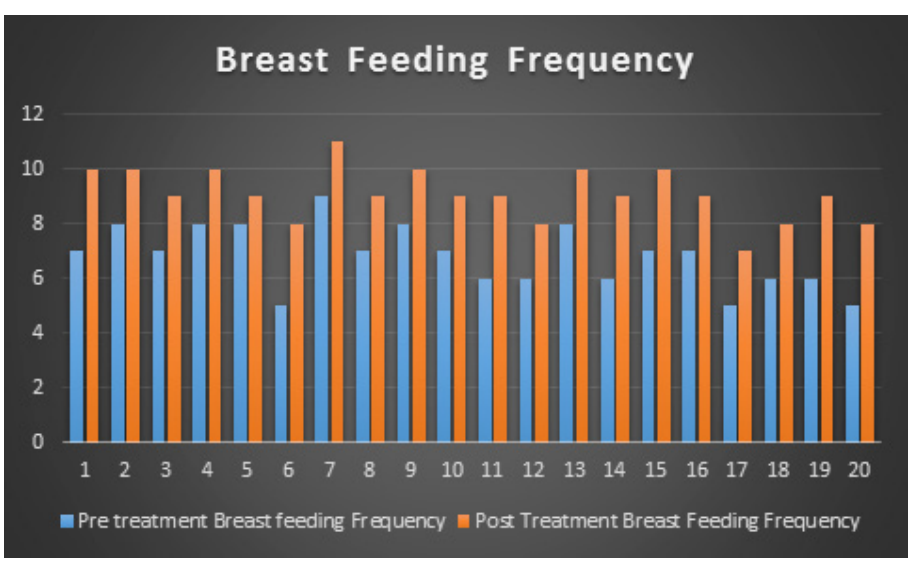

Graph 2. Comparison of breast feeding frequency graph.

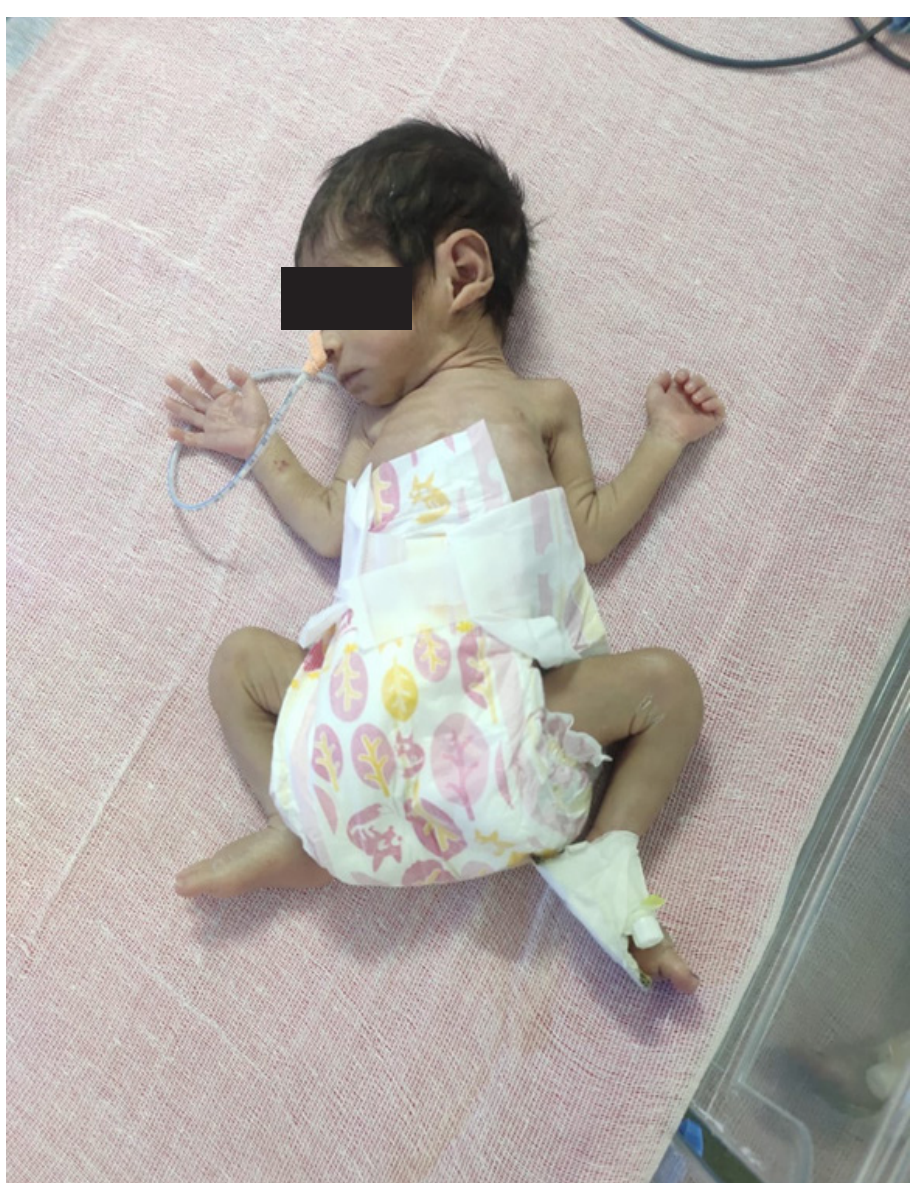

\subsection{Interpretation}

The below Graph 3 shows the comparison in post treatment weight measurement between three groups of preterm i.e. Moderately preterm, very preterm and extremely preterm group. In this we can see that moderately preterm group has more improvement in weight gain compare to very and extremely preterm neonates. 


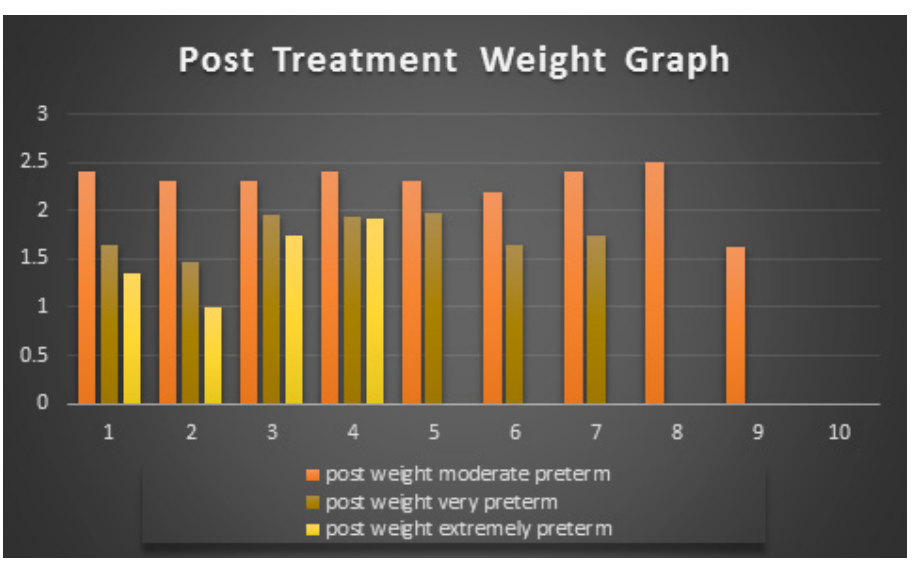

Graph 3. Post treatment weight comparative graph.

\subsection{Interpretation}

In the Graph 4 there is comparison in post treatment breast feeding frequency between moderately preterm, very preterm and extremely preterm neonates. In this graph we can see that moderately preterm neonates have slight more improvement in breast feeding frequency than extremely preterm neonates. But there is not much significant difference in post treatment breast feeding frequency of three groups.

\section{Discussion}

The purpose of this study was to find out the effectiveness of early physiotherapy interventions for weight gain in low birth weight neonates. Birth rate of premature babies has been increasing in last many years. But there is decrease in mortality rate of premature neonates due to advances in the field. Most attention must be given to the growth of premature neonates. In early months neonates explore most of the world by touching, the touch may be active or passive. Passive touch should be soft and caring and can be delivered by kangaroo mother care, handling and also massage. Massage gives a tactile stimulation which helps in growth and weight gain in LBW neonates. In this along with massage we have given proprioception stimulation, passive movements and kangaroo mother care.

In this study we have taken total20 premature LBW neonates. In which $15 \%$ were below $1 \mathrm{~kg}, 50 \%$ were between $1-2 \mathrm{~kg}$ and $35 \%$ were above $2 \mathrm{~kg}$. Preterm birth causes variety of problems, from that the low birth weight and postnatal morbidity are main. Early intervention consists of multidisciplinary services provided to baby right from his birth to promote baby health, minimizing the developmental delays and prevent functional deterioration.

In the study done by Priya Singh and Rangey and Megha Sheth it was shown that the massage and kangaroo mother care both were equally effective in improving neonate's weight and

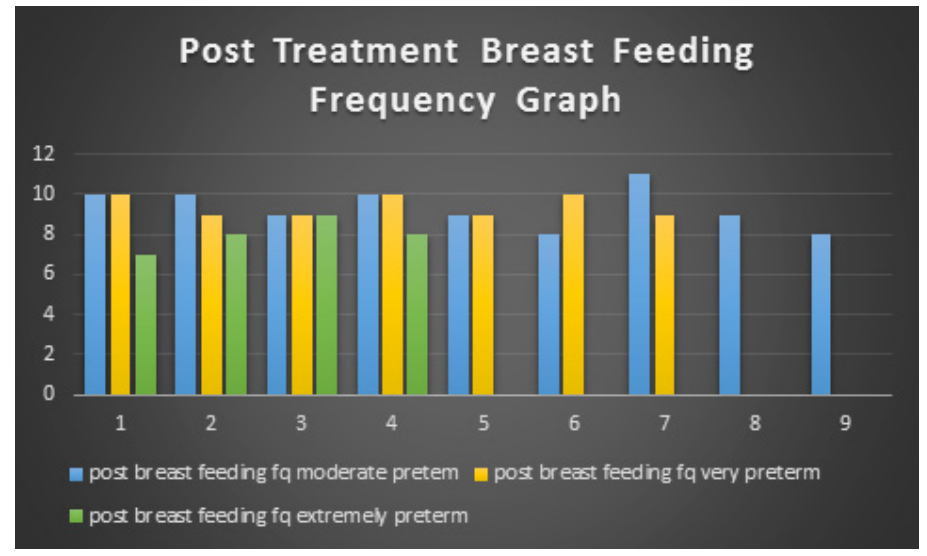

Graph 4. Post treatment breast feeding frequency comparison graph.

also for reducing hospital stay. Our study supports this article as in our study both the massage and kangaroo mother care has been used to treat neonates for weight gain and has proven to be effective in preterm neonates ${ }^{2}$.

However the proprioceptive stimulation by joint compression and swaddling has found to have secondary effect of calming baby and influences positive neurobehavioral state $^{5}$. Passive movements have also found to be effective for increasing weight and for emphasising correct movement pattern in baby. These techniques are known also to improve nutrition and mineralisation of bones by movements.

Oral stimulation helps in improving sucking response of neonate which increases the breast feeding frequency of baby which in-turn helps neonate to gain weight.

In study done by Paresh kumar Thakkar on neonates less than 32 weeks, he made two groups in group A multisensory stimulations were given and in group B specific physiotherapy interventions were given by massage, kinesthetic exercises and oral stimulation. He found that specific physiotherapy intervention group had better sucking coordination than group A. Hence, oral stimulation gives better sucking coordination and helps in weight gain of preterm neonates ${ }^{11}$.

In this study we also found out that the parental education and coordination is most important while treating babies in NICU. However one can do further study with larger sample size and with long-term follow up of neonates for any complications or delayed milestones.

\section{Conclusion}

On basis of result it can be concluded that the early physiotherapy is effective in improving weight, breast feeding frequency in low birth weight neonates. Hence, the early physiotherapy interventions should be considered to decrease the morbidity and mortality rate of LBW neonates. 


\section{Conflict of Interest}

The authors declare that there are no conflicts of interest concerning the content of present study.

\section{Source of Funding}

Self-funded

\section{Ethical Clearance}

The ethical clearance was obtained from Krishna institute of medical science ethical committee and the protocol number is 297/2019-2020.

\section{Abbreviations}

LBW- Low Birth Weight

NICU- Neonatal Incentive Care Unit

KMC- Kangaroo Mother Care

\section{Acknowledgment}

I praise and thank GOD with all my heart for his constant love, protection, guidance and blessings throughout my study. I would like to express my most humble and profound gratitude to respected Dean Dr. G. Varadharajulu, faculty of physiotherapy, KIMSDU for his inspiration, motivation, valuable guidance and suggestions throughout this project.

I wish to express my sincere thanks to Dr. S. Kakade and Mr. D. Mane sir for helping to carry out statistical analysis.

I would also like to express my sincere thanks to Dr. Namrata Kadam, faculty of pediatric physiotherapy, KIMSDU, all teaching and non-teaching staff of faculty of physiotherapy, KIMSDU, for their valuable suggestions and guidance.

Prof. and HOD Dr. C.D. Aundhakar pediatric department KIMSDU, Karad and Prof. and HOD Dr. Neeta Hatkar pediatric department SBHGMC, Dhule.

Above all with love I thank my grandparents, parents, and batch mates for their love, care and continuous encouragement. hospital stay in low birth weight preterm infants. Int. J. Pediatr. 2014; 2014:434060. https://doi.org/10.1155/2014/434060. PMid: 24976830, PMCid: PMC4058189.

3. Rad Zahraakbarian, Mojaveri, Mohsen, Javadian, Yahya, Hajiahmadi, Mahmoud, Kazemian, Fahimeh. The effect of massage on weight gain in very low birth weight neonates. Journal of Clinical Neonatology. 2016; 5:96. https://doi.org/10.4103/22494847.179900

4. Weerapong $\mathrm{P}$, Hume $\mathrm{Pa}$, Kolt Gs. The mechanisms of massage and effects on performance, muscle recovery and injury prevention. Sports Med. 2005; 35(3):235-56. https://doi. org/10.2165/00007256-200535030-00004. PMid: 15730338.

5. Anderson J. Sensory intervention with the preterm infant in the neonatal intensive care unit. Am. J. Occup. Ther. 1986; 40(1):19-26. https://doi.org/10.5014/ajot.40.1.19. PMid: 2418686.

6. Sharma Neha, Samuel Asir, Aranha Vencita. Pediatric physiotherapists' role in the neonatal intensive care unit: parent and health-care providers' perspectives. Journal of Clinical Neonatology. 2018; 7:111. https://doi.org/10.4103/jcn. JCN 2618.

7. Cameron Emma C, Maehle Valerie, Reid, Jane, Dip. The effects of an early physical therapy intervention for very preterm, very low birth weight infants: a randomized controlled clinical trial. Pediatric Physical Therapy. 2005; 17(2):107-19 https://doi. org/10.1097/01.PEP.0000163073.50852.58. PMid: 16357661.

8. Suraj Gupte. The Short Textbook of Pediatrics. Jaypee the Health Sciences Publisher, New Delhi; 2020.

9. Newman LF. Social and sensory environment of low birth weight infants in a special care nursery. An anthropological investigation. The Journal of Nervous and Mental Disease. 1981; 169(7):448-55. https://doi.org/10.1097/00005053-19810700000006. PMid: 7241110.

10. Jane Case Smith. An efficacy study of occupational therapy with high-risk neonates. American Journal of Occupational Therapy. 1988; 42(8):499-506. https://doi.org/10.5014/ajot.42.8.499. PMid: 3228153.

11. Nadar Unis, Thakkar Paresh Kumar, Shah Chaitali. Effect of specific physiotherapy interventions in comparison with multisensory stimulation on neurobehavioral outcome and weight gain in preterm infants: A randomized control trial. International Journal of Contemporary Pediatrics. 2017; 5:178. https://doi. org/10.18203/2349-3291.ijcp20175582.

\section{References}

1. Ramesh Agarwal, Vinod K Paul, Ashok K. Deorari essential pediatrics, New Delhi, CBS publishers and distributor; 2017.

2. Rangey PS, Sheth M. Comparative effect of massage therapy versus kangaroo mother care on body weight and length of 